Supporting Information for

\title{
Synthesis, Characterization, and Reactivity of New Copper(II) Complexes of 2-Methylthio- $N$-(2-pyridylmethyl)acetamide
}

\author{
Eric L. Klein, Masood A. Khan, and Robert P. Houser* \\ Department of Chemistry and Biochemistry, University of Oklahoma, 620 Parrington \\ Oval, Norman, Oklahoma 73019
}

General: All reagents were purchased from commercial sources and used without further purification. Solvents were dried and purified under nitrogen by using standard methods and were distilled immediately before use. GC/MS analyses are performed on a Thermoquest GCQ (ThermoFinnigan, San Jose, CA) mass spectrometer system using an electron impact (EI) as the ionization source $(70 \mathrm{eV}$ with the ion source temperature at $200{ }^{\circ} \mathrm{C}$ ), injector temperature is set at $220^{\circ} \mathrm{C}$ and a splitless mode of injection is used in all analyses. GC temperature gradient is set at $50{ }^{\circ} \mathrm{C}$ holding $5 \mathrm{~min}$, then at $20{ }^{\circ} \mathrm{C} / \mathrm{min}$ to increase to 250 holding for $20 \mathrm{~min} .{ }^{1} \mathrm{H}$ and ${ }^{13} \mathrm{C}$ NMR spectra were measured on a Varian $300 \mathrm{MHz}$ spectrometer using solvent as an internal standard. Electronic absorption spectra were measured on a Shimadzu UV2401PC UV-Vis spectrophotometer. IR spectra were recorded on a NEXUS 470 FTIR spectrometer. X-band EPR spectra were recorded on a Bruker EMX EPR spectrometer at $115 \mathrm{~K}$, a microwave frequency of $9.43 \mathrm{GHz}$, power set to $6.4 \mathrm{~mW}$, modulation frequency of $100 \mathrm{kHz}$, and modulation amplitude of 1.00 G. Elemental analyses were carried out by Atlantic Microlabs, Norcross, GA.

Synthesis of 2-HL ${ }^{\mathrm{N} 2 \mathrm{~S}}$ : DCC $\cdot \mathrm{H}_{2} \mathrm{O}(2.33 \mathrm{~g}, 11.3 \mathrm{mmol})$ dissolved in a minimal volume of THF was added in one portion to a $-10{ }^{\circ} \mathrm{C}$ THF solution of methylthioacetic acid $(1.00 \mathrm{~g}$, $9.42 \mathrm{mmol}$ ), HOBt (1.53 g, $11.3 \mathrm{mmol})$, and 2-(aminomethyl)-pyridine (1.02 g, 9.43 $\mathrm{mmol}$ ). The resulting mixture was stirred at $-10{ }^{\circ} \mathrm{C}$ for $1 \mathrm{~h}$, allowed to warm to room temperature, then stirred for $15 \mathrm{~h}$. The DCU precipitate was removed by filtration, the filtrate was concentrated and the product distilled under reduced pressure $\left(170{ }^{\circ} \mathrm{C}, 0.18\right.$ mmHg). Yield: $1.64 \mathrm{~g}$ (90\%). Anal. Calcd for $\mathrm{C}_{9} \mathrm{H}_{12} \mathrm{~N}_{2} \mathrm{OS}$ : C, 55.08; H, 6.16; N, 14.27. Found: C, 54.85; H, 6.54; N, 13.71. ${ }^{1} \mathrm{H} \mathrm{NMR}\left(\mathrm{CDCl}_{3}, 300 \mathrm{MHz}\right): \delta 8.48(\mathrm{~d}, J=4.7 \mathrm{~Hz}$, $1 \mathrm{H}), 7.96(\mathrm{~s}, 1 \mathrm{H}), 7.56-7.66(\mathrm{~m}, 1 \mathrm{H}), 7.10-7.26(\mathrm{~m}, 2 \mathrm{H}), 4.55(\mathrm{~d}, J=5.22 \mathrm{~Hz}, 2 \mathrm{H})$, $3.22(\mathrm{~s}, 2 \mathrm{H}), 2.11(\mathrm{~s}, 3 \mathrm{H}) \mathrm{ppm} .{ }^{13} \mathrm{C}\left\{{ }^{1} \mathrm{H}\right\} \mathrm{NMR}: \delta 168.47,156.02,148.78,136.48,122.13$, $121.66,44.53,37.95,16.29 \mathrm{ppm}$. 
Synthesis of 1: A methanol solution of $2-\mathrm{HL}^{\mathrm{N} 2 \mathrm{~S}}(250 \mathrm{mg}, 1.27 \mathrm{mmol})$ was added to a methanol solution of $\mathrm{CuCl}_{2} \cdot 2 \mathrm{H}_{2} \mathrm{O}(197 \mathrm{mg}, 1.15 \mathrm{mmol})$. The product was precipitated and isolated at low temperature, redissolved in warm methanol and recrystallized from $\mathrm{MeOH} / \mathrm{Et}_{2} \mathrm{O}$. Yield: $187 \mathrm{mg}(49 \%)$. Anal. Calcd for $\mathrm{C}_{9} \mathrm{H}_{12} \mathrm{Cl}_{2} \mathrm{CuN}_{2} \mathrm{OS}$ : C, 32.69; H, 3.66; $\mathrm{Cl}, 21.44 ; \mathrm{N}, 8.47$. Found: $\mathrm{C}, 32.38 ; \mathrm{H}, 3.72 ; \mathrm{Cl}, 21.57 ; \mathrm{N}, 8.36$. UV-visible $\left[\mathrm{H}_{2} \mathrm{O}, \mathrm{nm}\right.$ $\left(\mathrm{M}^{-1} \mathrm{~cm}^{-1}\right)$ ]: 656 (140). FTIR (KBr): 3107, 3068, 3045, 2956, 2914, 2749, 2661, 2463, 2295, 1685, 1642, 1608, 1570, 1493, 1480, 1437, 1441, 1426, 1397, 1374, 1360, 1326, $1282,1228,1160,1084,1054,1026,993,966,844,786,719,646 \mathrm{~cm}^{-1}$.

Synthesis of 2: A methanol solution of $\mathrm{L}^{\mathrm{N} 2 \mathrm{~S}}(250 \mathrm{mg}, 1.27 \mathrm{mmol})$ was added to a methanol solution of $\mathrm{CuCl}_{2} \cdot 2 \mathrm{H}_{2} \mathrm{O}(197 \mathrm{mg}, 1.15 \mathrm{mmol})$ and triethylamine (349 $\mathrm{mg}, 3.45$ $\mathrm{mmol}$ ). The product was precipitated and isolated at low temperature, dissolved into a minimal amount of methanol, and recrystallized from $\mathrm{MeOH} / \mathrm{Et}_{2} \mathrm{O}$. Yield: $127 \mathrm{mg}$ (38.8\%). Anal. Calcd for $\mathrm{C}_{10} \mathrm{H}_{15} \mathrm{ClCuN}_{2} \mathrm{O}_{2} \mathrm{~S}: \mathrm{C}, 36.81 ; \mathrm{H}, 4.63 ; \mathrm{Cl}, 10.87 ; \mathrm{N}, 8.59$. Found: C, 36.92; H, 4.42; Cl, 11.11; N, 8.82.UV-visible [MeOH, nm $\left.\left(\mathrm{M}^{-1} \mathrm{~cm}^{-1}\right)\right]: 642$ (240). EPR $\left(9.4339 \mathrm{GHz}, 1: 1 \mathrm{CH}_{2} \mathrm{Cl}_{2} / \mathrm{MeOH}, 120 \mathrm{~K}\right) g_{\|}=2.187, A_{\|}(\mathrm{Cu})=178 \times 10^{-4} \mathrm{~cm}^{-1}, g_{\perp} \approx 2.03$. FTIR (KBr): 3144, 2871, 2806, 1593, 1564, 1483, 1409, 1382, 1345, 1315, 1283, 1227 , 1212, 1162, 1089, 1051, 1026, 968, 881, 764, 719, 673, 646, 469, 418, 352, 310, 251, $216,205 \mathrm{~cm}^{-1}$. 


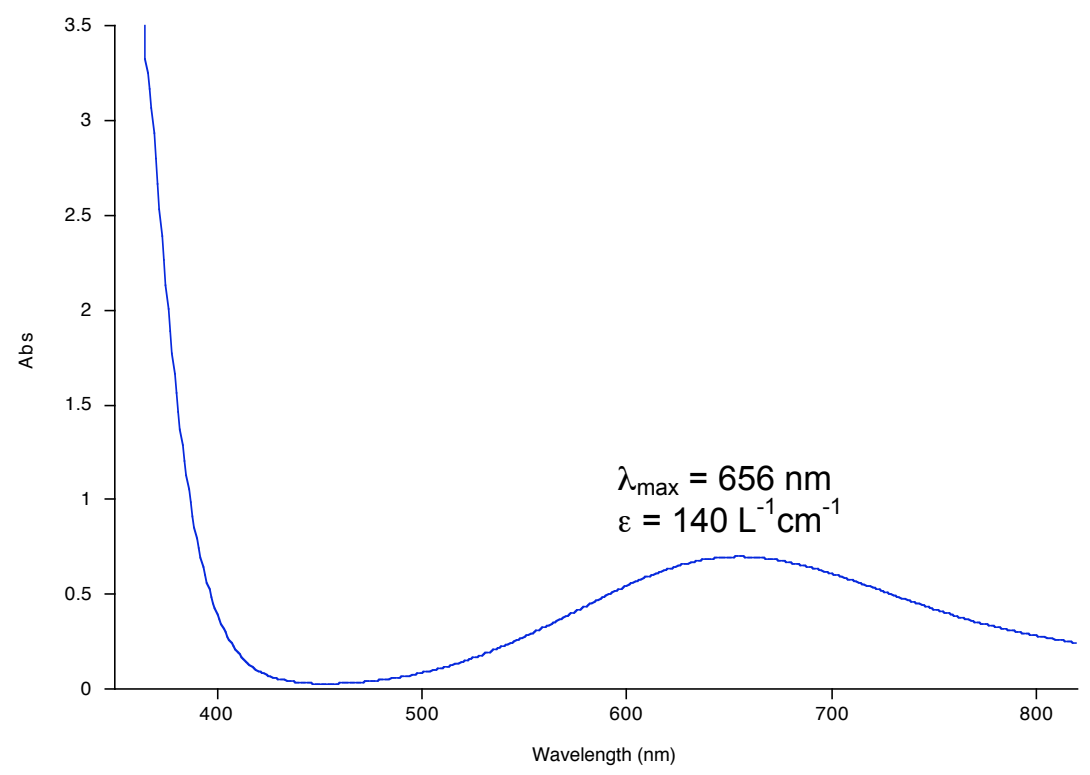

Figure S1. UV/visible spectrum of $\mathbf{1}$ in $\mathrm{H}_{2} \mathrm{O}$.

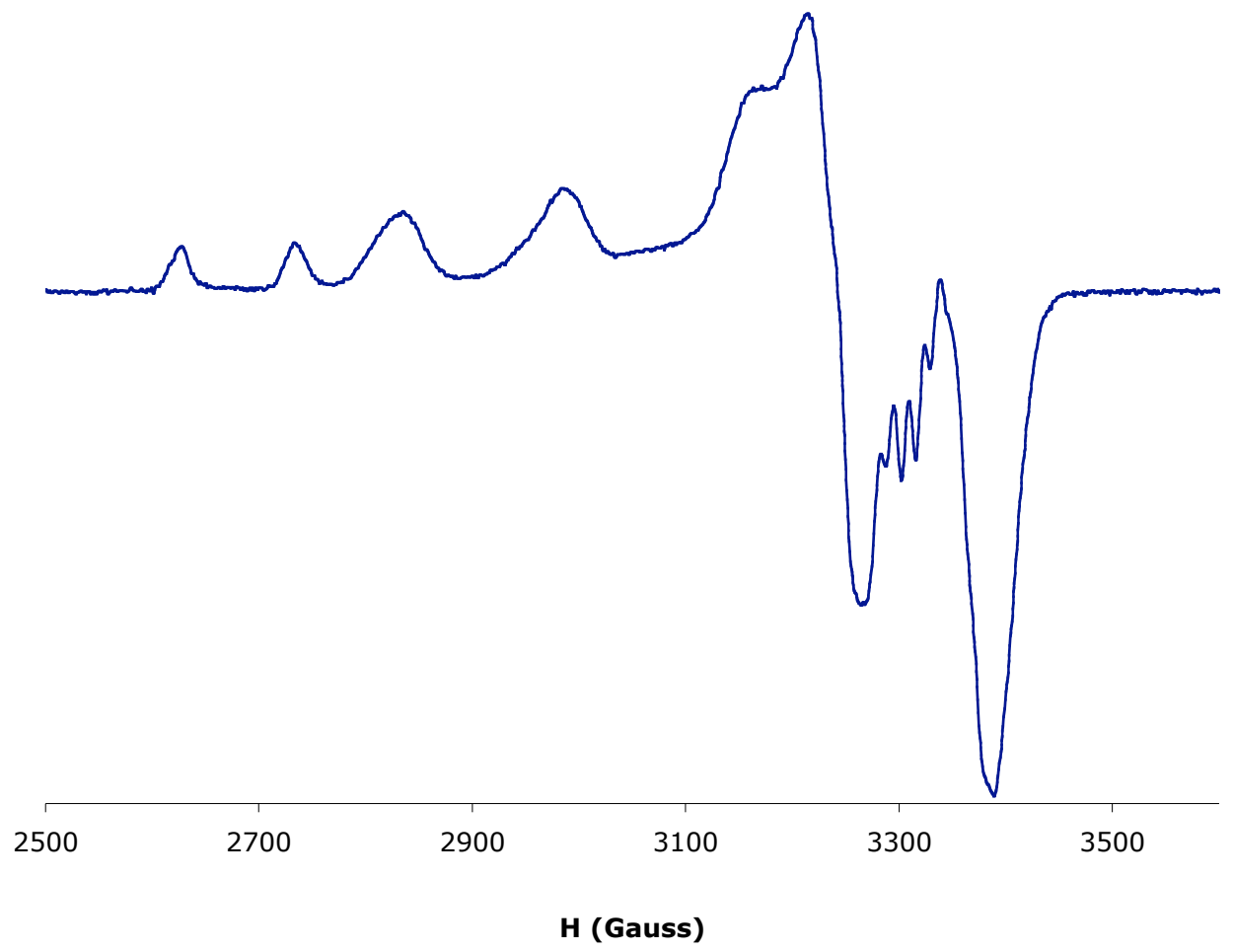

Figure S2. EPR spectrum of 1 in 10:5:4:1 EtOH/MeOH$/ \mathrm{CH}_{2} \mathrm{Cl}_{2} / \mathrm{H}_{2} \mathrm{O}$ at $120 \mathrm{~K}$. 


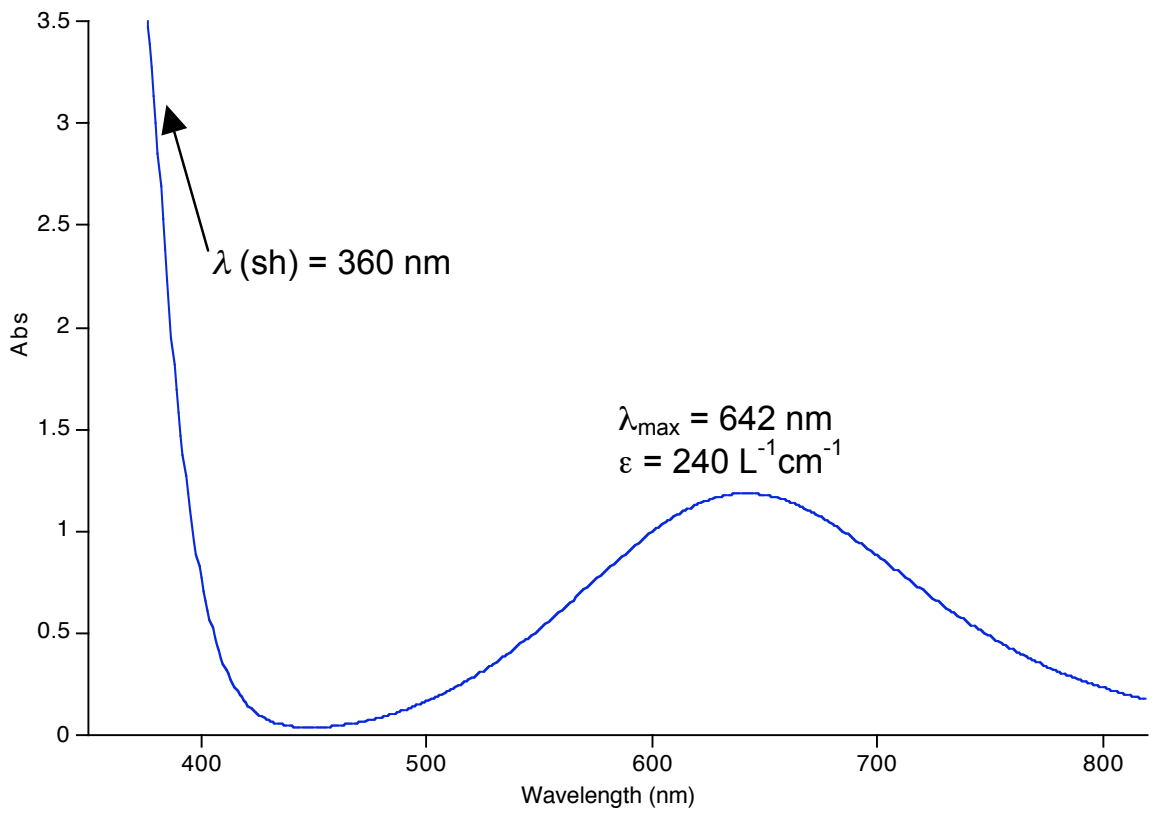

Figure S3. UV/visible spectrum of $\mathbf{2}$ in methanol.

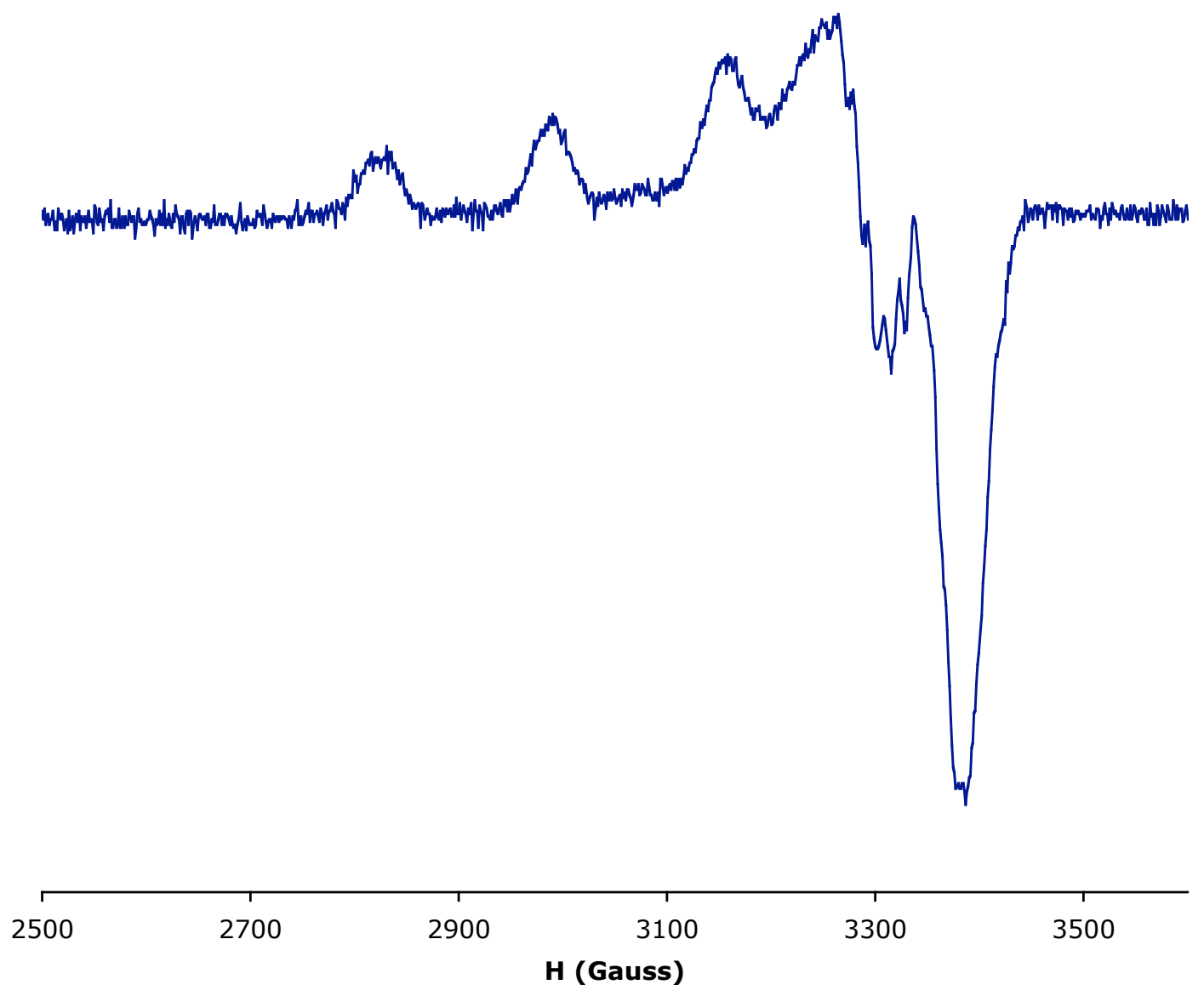

Figure S4. EPR spectrum of 2 in $1: 1 \mathrm{CH}_{2} \mathrm{Cl}_{2} / \mathrm{MeOH}$ at $120 \mathrm{~K}$. 


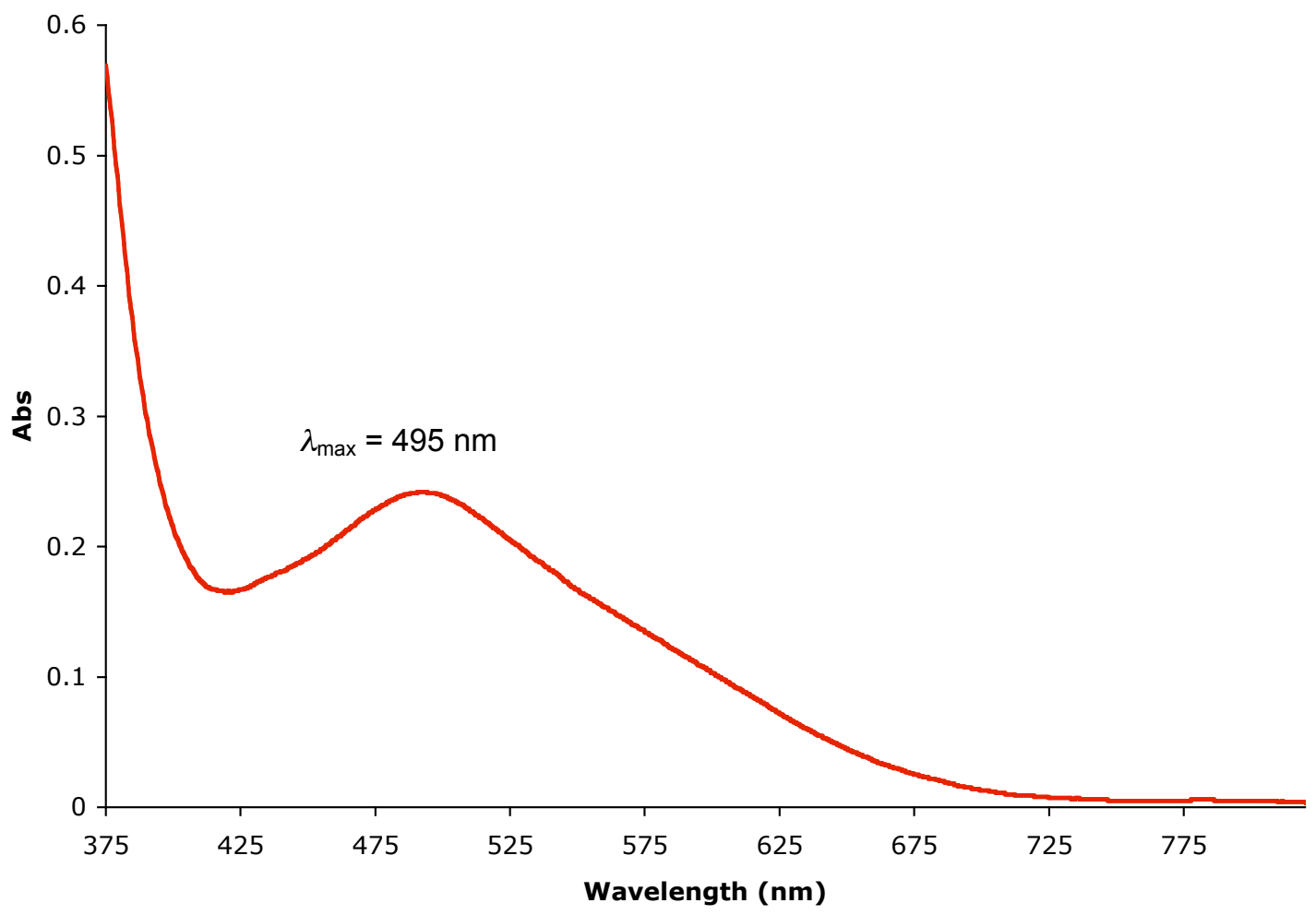

Figure S5. UV/visible spectrum of $\mathbf{2}(\mathrm{SAr})$ in dichloromethane.

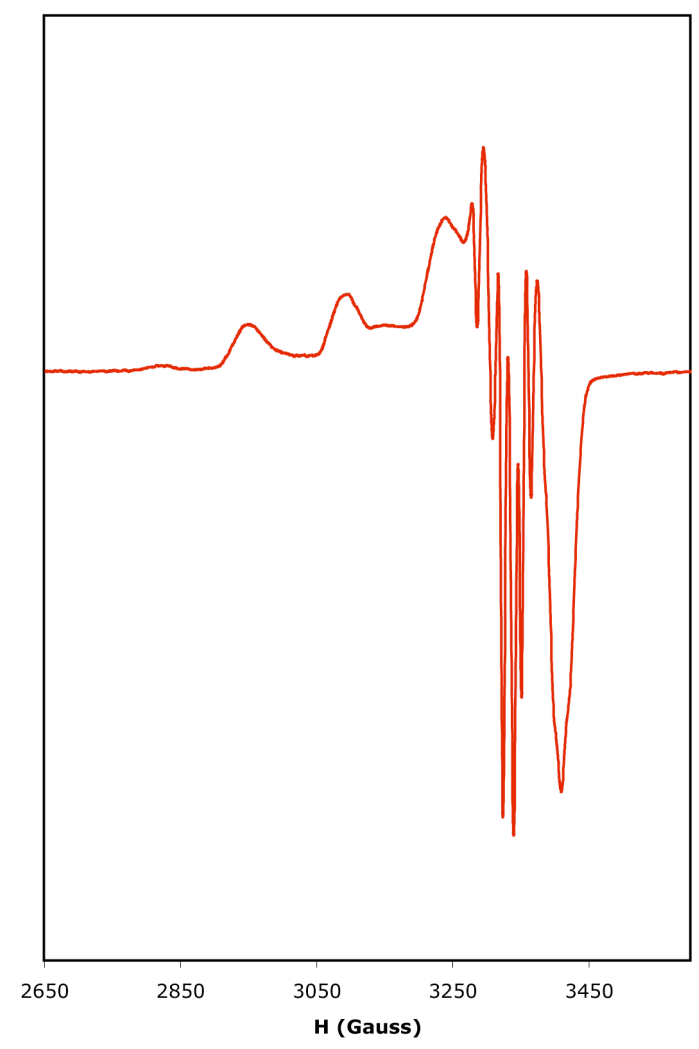

Figure S6. EPR spectrum of $2(\mathrm{SAr})$ in $\mathrm{CH}_{2} \mathrm{Cl}_{2} /$ toluene at $115 \mathrm{~K}$. 


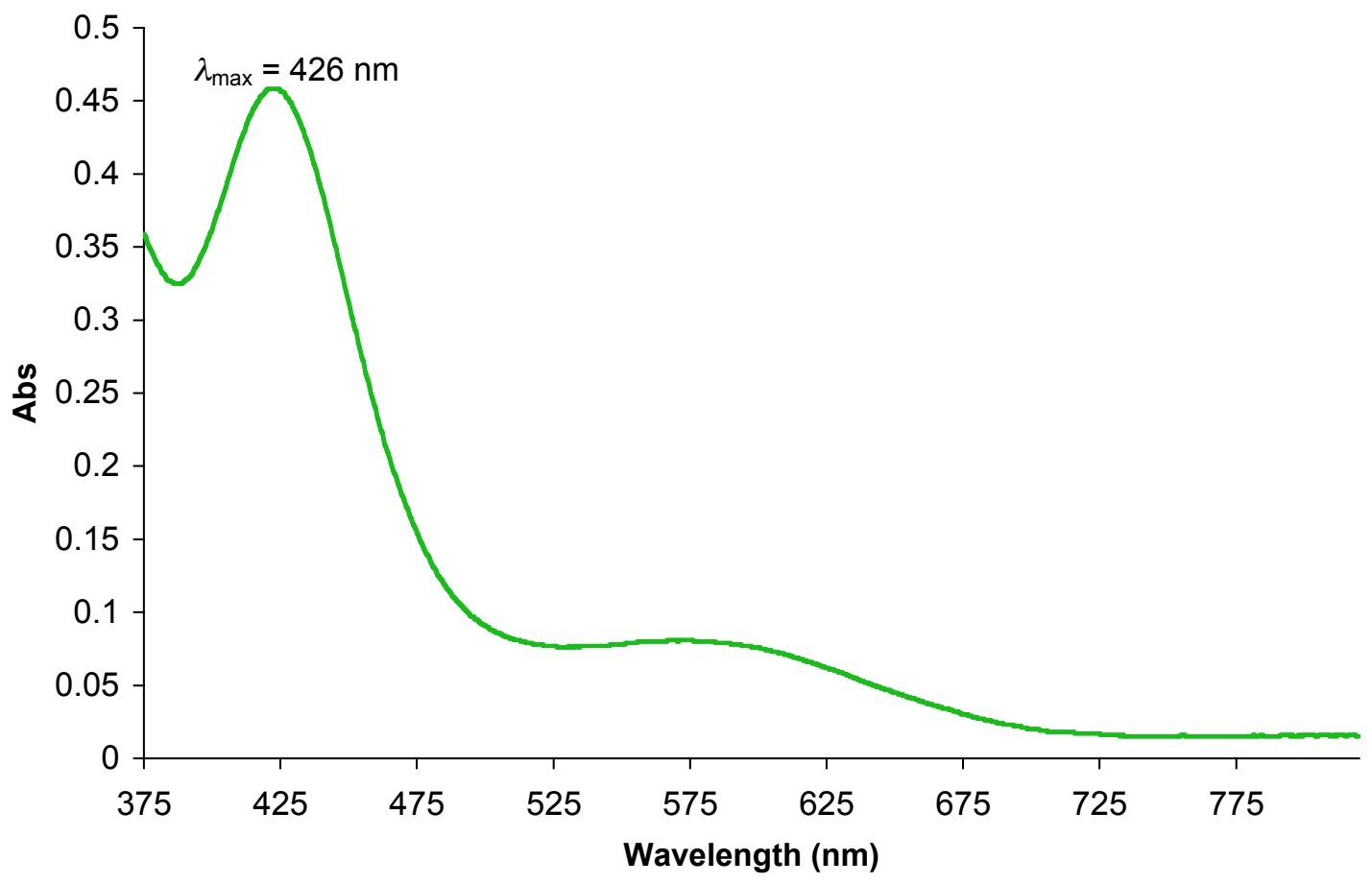

Figure S7. UV/visible spectrum of $\mathbf{2}\left(\mathrm{SCPh}_{3}\right)$ in dichloromethane.

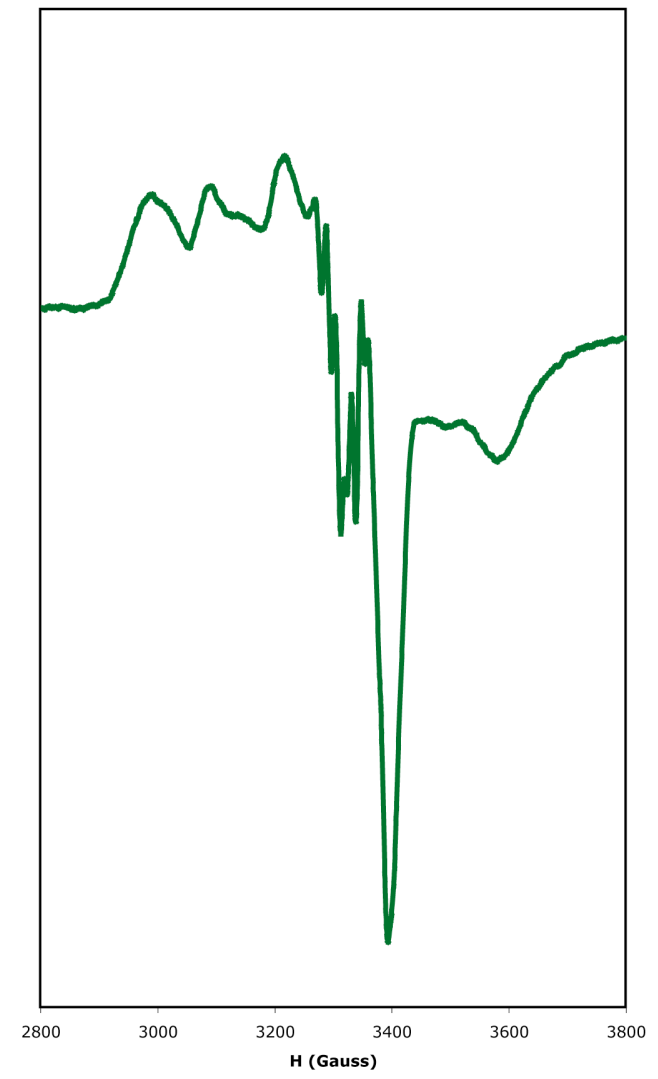

Figure S8. EPR spectrum of $2\left(\mathrm{SCPh}_{3}\right)$ in $\mathrm{CH}_{2} \mathrm{Cl}_{2} /$ toluene at $115 \mathrm{~K}$. 